Introduction Increasing human immunodeficiency virus (HIV) testing in South Africa is vital for the HIV response. Targeting young people is important as they become sexually active and because HIV risk rapidly increases as youth enter their 20s. This study aims to increase the understanding of high school learners' preferences regarding the characteristics of HIV testing service delivery models and to inform policy makers and implementers regarding potential barriers to and facilitators of HIV testing.

Methods An attitudinal survey was used to examine HIV testing prefer- ences among 248 high school learners in KwaZulu-Natal. Statistical tests were used to identify the most favoured characteristics of testing service delivery models and examine key differences in preferences based on demographic characteristics and testing history.

Results Most learners were found to prefer testing offered at a clinic on a Saturday (43\%), using a finger prick test (59\%), conducted by a doctor (61\%) who also provides individual counselling $(60 \%)$. Shorter testing times were preferred, as well as a monetary incentive to cover any associ- ated expenses. Time, location, the type of test, and who conducts the test were most important. However, stratified analysis suggests that preferences diverge, particularly around gender, grade, but also sexual history and previous testing experience. Conclusion Human immunodeficiency virus testing services can be improved in line with preferences, but there is no single optimal design that caters to the preferences of all learners. It is unlikely that a "one-size-fits-all" approach will be effective to reach HIV testing targets. A range of options may be required to maximise coverage.

\section{P4.64 ATTITUDINAL AND BEHAVIOURAL DIFFERENCES BETWEEN YOUTH WHO HAVE HAD ANAL SEX AND THOSE WHO HAVE NOT IN CAPE TOWN, SOUTH AFRICA}

${ }^{1}$ Michele Ybarra, ${ }^{1}$ Myeshia Price-Feeney, ${ }^{2}$ Kelvin Mwaba. ${ }^{1}$ Centre for Innovative Public Health Research, San Clemente, USA; ${ }^{2}$ Univeristy of Western Cape, Cape Town, South African Republic

\subsection{6/sextrans-2017-053264.560}

Introduction Although HIV rates among South African youth are among the highest in the world, most research efforts have overlooked anal sex as a contextualising factor.

Methods In 2012, a pencil-and-paper survey was completed by 937 youth 16 years of age and older who were attending low-income secondary schools in Cape Town.

Results Eleven and $31 \%$ of female and male youth, respectively, reported ever having anal sex. Among sexually active male and female respondents, those who had ever had anal sex were more likely to report inconsistent condom use, perpetrate dating violence, and experience victimisation and perpetration of sexually coercive behaviour. Female respondents who had ever had anal sex had significantly lower levels of HIV information than sexually active females who had not had anal sex.

Conclusion Rates of anal sex are higher for boys than girls in the survey, yet the associated risk factors were strikingly similar. Harm reduction strategies for anal sex should be made available to South African youth.

\section{P4.65 LESSONS LEARNED USING FB TO RECRUIT LGBT ADULTS ACROSS EASTERN AFRICA INTO ONLINE SEXUAL HEALTH FOCUS GROUPS}

Michele Ybarra, Emilie Chen, Tonya Prescott. Centre for Innovative Public Health Research, San Clemente, USA

\subsection{6/sextrans-2017-053264.561}

Introduction Because of cultural and legal discrimination enacted towards LGBT people in eastern Africa, innovative methods are needed to safely engage LGBT Africans in sexual health research.

Methods We conducted two online focus groups (FGs) with eastern African MSM, one in December 2016 and the other in January 2017. Participants were recruited via Facebook advertisements, which were targeted to men who: were 'interested in' men or were 'interested in' men and women; were living in: Rwanda, Uganda, Kenya, Tanzania, or South Sudan; and were 18 years and older. People who clicked on the ad were linked to the study website, which explained the FG activity, provided an eligibility screener, and a consent form for those who were eligible. Once consented, MSM were linked to the online FG, where they could anonymously post answers.

Results After a 27 hour FB campaign, 176480 people were reached across eastern Africa, 11889 of whom clicked on the ad. Most clicks (59\%) were from people in Tanzania; Kenya (22\%) and Uganda (13\%) were the next most common sources of clicks. Thirty-three people completed a screener and were eligible, and 22 consented to take part in the first FG. Of those who consented, 5 people posted in at least one thread in the online FG bulletin board. Of the 15 threads we posted, 3 received posts from participants, none of which were sexual health-related. To invigorate response rates, the FG script was shortened from 15 to 6 threads and displayed on one instead of three separate pages. A second 27 hour FB campaign was launched. A similar number of eligible people were identified $(n=35)$ while more consented $(n=34)$. One person went on to post in 4 sexual health-related threads, and another posted in one.Additional FGs are being conducted. Lessons learned that can inform future research that endeavours to engage LGBT Africans will be presented.

Conclusion Although online data collection holds promise in reaching hidden populations, piloting work is necessary to determine the most effective way to ensure privacy and promote participation.

\section{P4.66 OPPORTUNITIES TO TAILOR HIV PREVENTION PROGRAMMING FOR UGANDAN YOUNG ADULTS}

${ }^{1}$ Michele Ybarra, ${ }^{1}$ Tonya Prescott, ${ }^{2}$ Emmanuel Kyagaba. ${ }^{~}$ Centre for Innovative Public Health Research, San Clemente, USA; ${ }^{2}$ Internet Solutions for Kids, Uganda; Mbarara University of Science and Technology, Mbarara, Uganda

\subsection{6/sextrans-2017-053264.562}

Introduction Uganda, like most Sub-Saharan African countries, has comprehensive HIV prevention campaigns, yet programs tailored to young adults are completely lacking.

Methods In 2016, 202 18-22 year olds were recruited from across Uganda via Facebook to take part in online, 3 day, asynchronous focus groups (FGs). The aim of the FGs was to understand sexual health decision making to inform HIV prevention content development. The study sample was 